\title{
A design science nas pesquisas em design no Brasil
}

\section{Design science in design researches in Brazil}

\author{
Iana Uliana Perez, UNESP - Universidade Estadual Paulista \\ iana.uli@gmail.com
}

\author{
Mônica Cristina de Moura, UNESP - Universidade Estadual Paulista \\ monica.moura@unesp.br
}

Fausto Orsi Medola, UNESP - Universidade Estadual Paulista

fausto.medola@unesp.br

\section{Resumo}

Este artigo apresenta a abordagem da design science como alternativa para as investigações em design e nas ciências sociais aplicadas em geral. Para a sua redação, foi empreendida Revisão Bibliográfica Sistemática no Catálogo de Teses e Dissertações da Capes, verificando-se a incidência dos termos "design science" e "action design research". O levantamento permitiu a identificação de 14 teses e dissertações em design que adotaram métodos próprios da design science, como Design Science Research (DSR) e Action Design Research (ADR). Para caracterizar esses métodos, é apresentada análise comparativa das pesquisas de três teses que adotaram a DSR; também é relatada a experiência de realização de uma pesquisa de mestrado que utilizou a ADR. A discussão ressalta as contribuições desses métodos e os aspectos que precisam ser aprimorados para sua operacionalização.

Palavras-chave: Pesquisa científica, Design Science Research, Action Design Research

\begin{abstract}
This article presents the design science approach as an alternative for design research and applied social sciences in general. For its writing, we carried out a systematic bibliographical review in the Capes Thesis and Dissertations Catalog, verifying the incidence of the terms "design science" and "action design research". The survey allowed the identification of 14 theses and dissertations in design that adopted design science methods, such as Design Science Research (DSR) and Action Design Research (ADR). To characterize these methods, this article presents a comparative analysis of the research of three theses that adopted the DSR; it also reports the experience of conducting a master's research that used ADR. The discussion highlights the contributions of these methods and the aspects that need to be improved for their operationalization.
\end{abstract}

Keywords: Scientific research, Design Science Research, Action Design Research 


\section{Introdução}

O presente artigo discute a importância da utilização de métodos de pesquisa baseados na design science, como a Design Science Research e a Action Design Research, no campo da pesquisa em design. Esses métodos se destacam por sua importância e aderência à produção de conhecimento em design, pois permitem-lhe o emprego de suas próprias perspectivas metodológicas. Assim, o estudo aqui apresentado tem como objetivo principal apresentar esses métodos próprios da design science como alternativas para a realização de pesquisas científicas em design. Os objetivos específicos são: identificar e caracterizar os métodos da design science; verificar a sua incidência nas pesquisas nacionais desenvolvidas no âmbito da pós-graduação stricto sensu; analisar como teses e dissertações em design brasileiras têm empregado esses métodos. Para atender aos objetivos, foi conduzida uma Revisão Bibliográfica Sistemática (RBS) no Catálogo de Teses e Dissertações da Capes.

A seguir, é apresentada reflexão teórica sobre a relação entre design, pesquisa e ciência, de modo a elucidar o lugar da design science nesse campo e discutir sobre a adequação dos métodos científicos tradicionais para as pesquisas na área do design. Na sequência, são explicitados os procedimentos metodológicos da RBS e os seus resultados, descritos. A discussão é dividida em duas partes: 1) apresentação da Design Science Research por meio da análise de três teses que a adotaram como método e 2) relato de experiência de uma dissertação que utilizou a Action Design Research.

\section{A pesquisa científica em design}

O design por muito tempo procurou aproximar-se das ciências, baseando seu processo projetual em procedimentos científicos de áreas consolidadas (BONSIEPE, 2011). O inverso, contudo, não ocorria até o advento, na década de 1990, de métodos científicos baseados na design science. Agora, são as "ciências" que procuram uma aproximação com o design para conduzir seus processos metodológicos. A design science apresenta como pressuposto a necessidade de uma ciência que se dedique à proposição e desenvolvimento de artefatos, entendidos como tudo aquilo que é construído pelo ser humano. É, portanto, uma ciência pragmática cuja finalidade é "conceber um conhecimento sobre como projetar, e não apenas aplicá-lo" (SANTOS, 2018; DRESCH; LACERDA; ANTUNES JÚNIOR, 2015, p. 57; SIMON, 1996).

Para elucidar as associações entre design e ciência, Cross (2011) propõe três diferentes interpretações: 1) design científico, 2) design science e 3) ciência do design. A primeira remete ao design moderno e às tentativas de basear os métodos de design no conhecimento científico. Já design science diz respeito a uma abordagem sistemática para o design, na qual o design é uma atividade científica em si. A ciência do design, por sua vez, refere-se às investigações que buscam melhorar o entendimento sobre design por meio de métodos científicos. Essas três interpretações assemelham-se à categorização da pesquisa em design proposta por Frayling (1993) e reinterpretada por Manzini (2015):

- Pesquisa sobre design - auxilia a entender a natureza do próprio design, envolvendo uma variedade de perspectivas teóricas de áreas diversas;

- Pesquisa para o design - produz melhores ferramentas operacionais e conceituais para a atividade de design; 
- Pesquisa através do design - adota métodos originais baseados em ferramentas e habilidades próprias da cultura e da prática do design.

Pode-se dizer que a pesquisa sobre design refere-se à ciência do design, enquanto a pesquisa para o design remete ao design científico e a pesquisa através do design, à design science. Assim, a pesquisa científica em design apresenta diferentes perspectivas quanto à relação entre design e ciência, podendo adotar abordagens distintas. Contudo, em situações de deslocamento de metodologias tradicionais de outras áreas para o design, "nem sempre a transposição dos conceitos ou métodos consegue manter o rigor ou a coerência teórica exigida pelas áreas de origem" (SILVA, 2015, p. 24). Embora os métodos tradicionais possam ser aproveitados na pesquisa em design, é importante que essa apropriação ocorra quando pertinente (CROSS, 2001), isto é, quando há compatibilidade com os objetivos da pesquisa. Caso contrário, a adaptação para o design de procedimentos de pesquisa provenientes de outros campos pode fazer com que "(a) as pesquisas de cunho descritivo dos projetos não possuam um prestígio acadêmico" ou (b) exigir "dos pesquisadores um distanciamento das questões relevantes à prática do projeto para a produção de um conhecimento científico tradicional" (SILVA, 2015, p. 24). Por isso, destaca-se, aqui, a design science como um paradigma epistemológico que permite ao design empregar suas próprias perspectivas metodológicas.

\section{Método}

Para investigar a utilização de métodos baseados na design science no âmbito da pós-graduação stricto sensu brasileira, adotou-se o método indutivo com abordagem quanti-qualitativa, caráter exploratóriodescritivo e natureza básica. O delineamento da pesquisa é a Revisão Bibliográfica Sistemática (RBS), seguida de relato de experiência. A RBS é uma pesquisa bibliográfica que segue um método explícito, planejado e justificável, de modo a apresentar foco e rigor científico (DRESCH; LACERDA; ANTUNES JÚNIOR, 2015; CONFORTO; AMARAL; SILVA, 2011). Para a sua aplicação, foi utilizado o roteiro de Conforto, Amaral e Silva (2011), dividido em três fases: Entrada, Processamento e Saída. Durante a fase de Entrada, foram definidos os aspectos apresentados no Quadro 1.

\begin{tabular}{rll}
\hline Base de dados & Catálogo de Teses e Dissertações da Capes. \\
\hline Strings & "design science” e “action design research" \\
\hline Data das buscas & Novembro de 2018, com atualização em maio de 2019 \\
\hline Critérios de inclusão & $\begin{array}{l}\text { Utilizar um método de pesquisa científica baseado na design science. } \\
\text { Pesquisa realizada em Programas de Pós-Graduação em Design no Brasil. }\end{array}$ \\
\hline Critérios de exclusão & $\begin{array}{l}\text { Dissertação de mestrado profissional. } \\
\text { Não obter acesso ao arquivo com a dissertação ou tese completa. }\end{array}$ \\
\hline Filtros adotados & $\begin{array}{l}\text { Filtro 1 - critérios de inclusão e exclusão: leitura do título e resumo. } \\
\text { Filtro } 2 \text { critério de qualificação: leitura da introdução, método, resultados }\end{array}$ \\
\hline Critério de qualificação & Qualidade da execução do método e acesso ao texto completo. \\
\hline
\end{tabular}

Quadro 1: Procedimentos metodológicos da RBS. Elaborado pelos autores. 
As buscas foram realizadas durante a segunda fase, Processamento, assim como a documentação e avaliação. A partir da leitura e análise dos trabalhos, foi elaborada a síntese dos resultados (Saída), que compõe a seção seguinte. Depois da apresentação dos resultados, são discutidas três teses que adotaram a Design Science Research e é relatada a experiência de aplicação de uma pesquisa-ação sob o paradigma da design science, método denominado por Sein et al. (2011) como Action Design Research. Trata-se da pesquisa de mestrado de uma das autoras deste artigo (PEREZ, 2018), realizada no Programa de Pós-Graduação em Design da Universidade Federal do Paraná, sob orientação do Prof. Dr. Aguinaldo dos Santos. O objetivo do relato é demonstrar a aplicação de um método híbrido baseado na design science e discutir com mais profundidade suas contribuições e os desafios encontrados durante a realização da pesquisa.

\section{Resultados}

A partir de busca no Catálogo de Teses e Dissertações da Capes, realizada em novembro de 2018 e atualizada em maio de 2019, verificou-se a ocorrência de 331 trabalhos para "design science" e dois para "action design research" (uma dissertação em design de 2018 e uma tese de 2015 em engenharia). A linha do tempo da Figura 1 sugere que há uma tendência crescente de adoção da design science; todavia, a imagem também mostra que poucas pesquisas sobre design science são realizadas em cursos de pós-graduação em design, que representam apenas 5,4\% dos trabalhos.
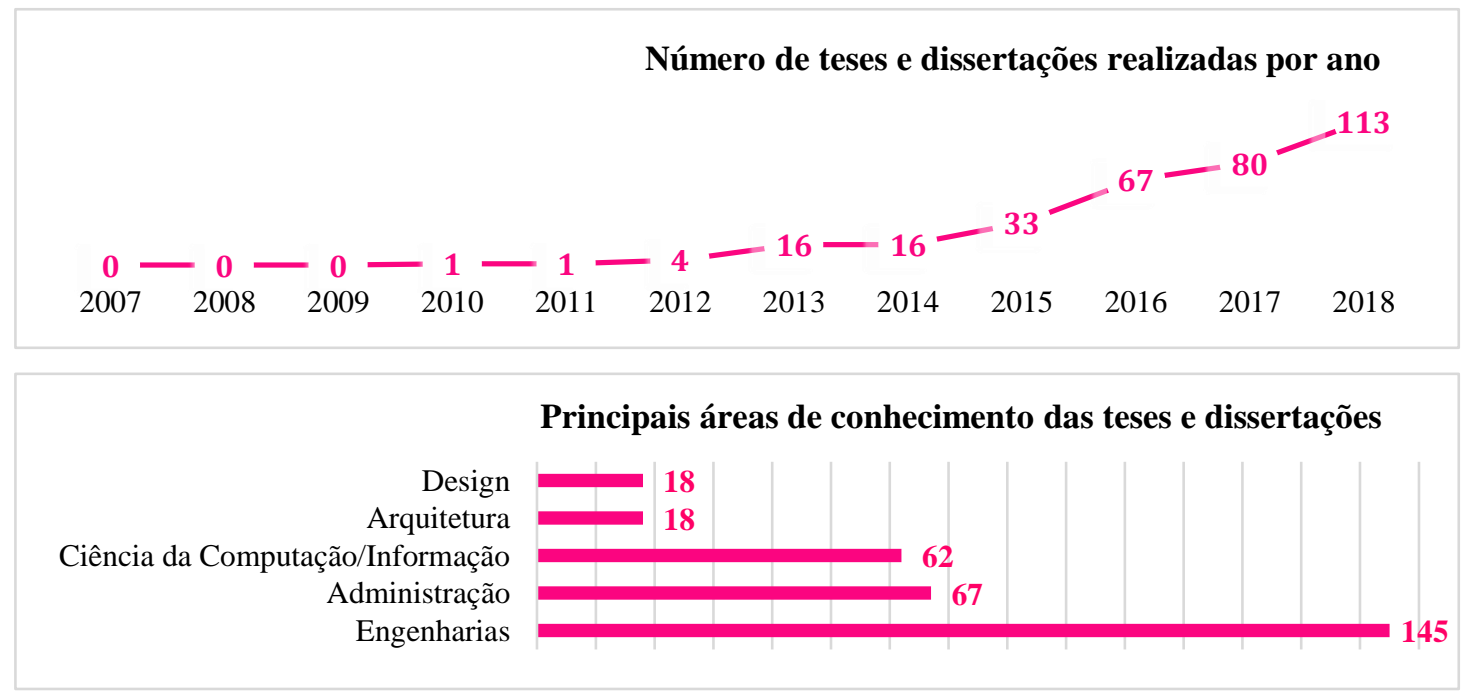

Figura 1: Resultados sobre design science em todas as áreas de conhecimento. Elaborada pelos autores.

Dos 18 resultados para design science na área de design, 14 foram selecionados após o filtro 1: oito teses e seis dissertações que utilizaram o método Design Science Research (DSR). Apenas três teses, contudo, foram selecionadas para análise (SMYTHE, 2018; TEIXEIRA, 2018; MESACASA, 2018), pois, quando a pesquisa aqui relatada foi realizada, foram as únicas que atendiam ao critério de qualificação. Destaca-se que, após seis meses, quando a busca foi atualizada, dobrou o número de trabalhos em design selecionados após o filtro 1. Quanto ao método Action Design Research 
(ADR), foi encontrada apenas uma dissertação em design, que será alvo do relato de experiência. $\mathrm{O}$ resumo dos resultados é apresentado na Figura 2.

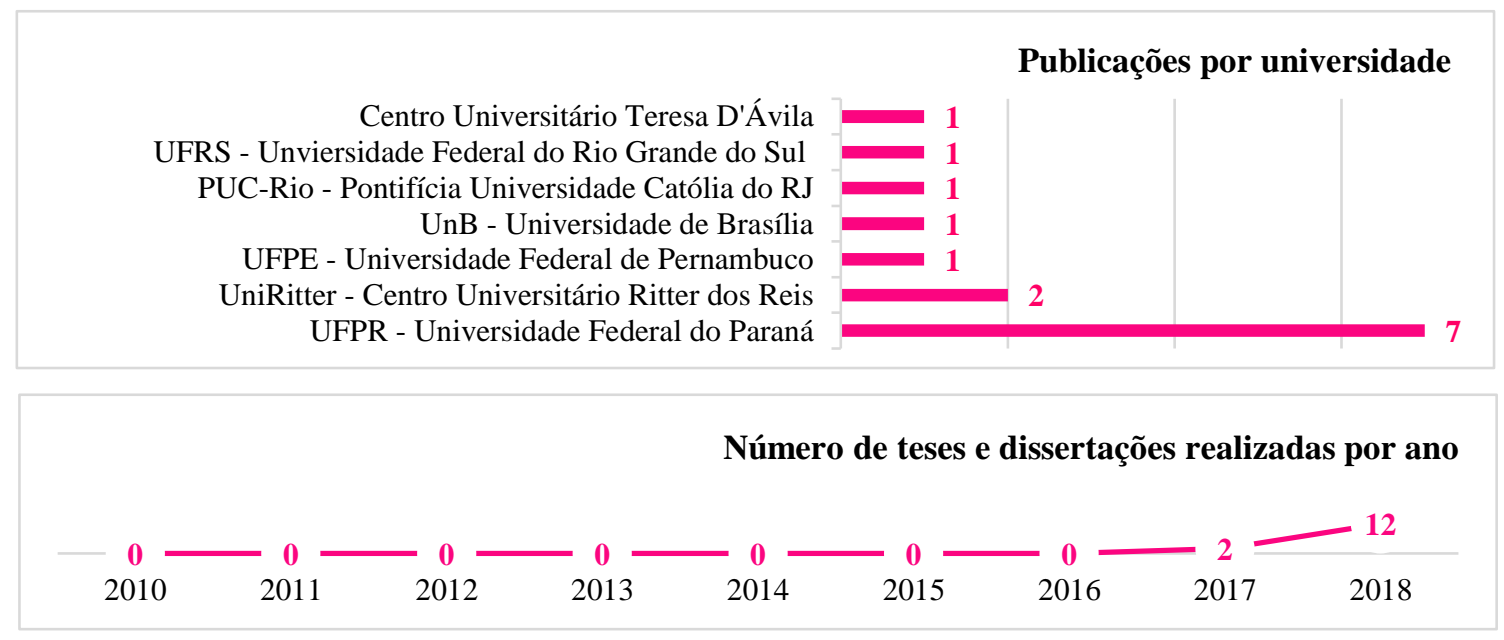

Figura 2: Resultados sobre design science na área de design. Elaborada pelos autores.

A linha do tempo mostra que as primeiras pesquisas foram realizadas em 2017. Como a primeira ocorrência da design science em teses e dissertações brasileiras de outras áreas data de 2010, é possível inferir que é tardia a adoção da design science por parte das pesquisas de pós-graduação em design. Contudo, o crescimento em 2018 sugere uma tendência de alteração desse cenário.

\section{Uso da Design Science Research nas pesquisas científicas em design}

As três teses aqui analisadas foram desenvolvidas no Programa de Pós-Graduação em Design da UFPR e defendidas em 2018. Todas tinham como objetivo desenvolver artefatos, o que justifica a escolha do método Design Science Research (DSR), pois ele é o mais indicado nesse caso, segundo Dresch, Lacerda e Antunes Júnior (2015). O artefato desenvolvido por Smythe (2018) foi o método CIW - Comportamento Informacional no Wayfinding ${ }^{l}$, para coleta de dados sobre o comportamento informacional dos usuários no processo de wayfinding em ambientes hospitalares. Teixeira (2018) também desenvolveu um método, denominado MIPOTI, mas voltado para a inclusão de pessoas com deficiência em postos de trabalho de produção industrial. Já Mesacasa (2018) projetou um modelo de processo de design de moda, nomeado DIM, que integra gestão do design e inovação. A representação gráfica desses três artefatos é apresentada na Figura 3, de cunho ilustrativo.

\footnotetext{
${ }^{1}$ Processo de orientação espacial.
} 

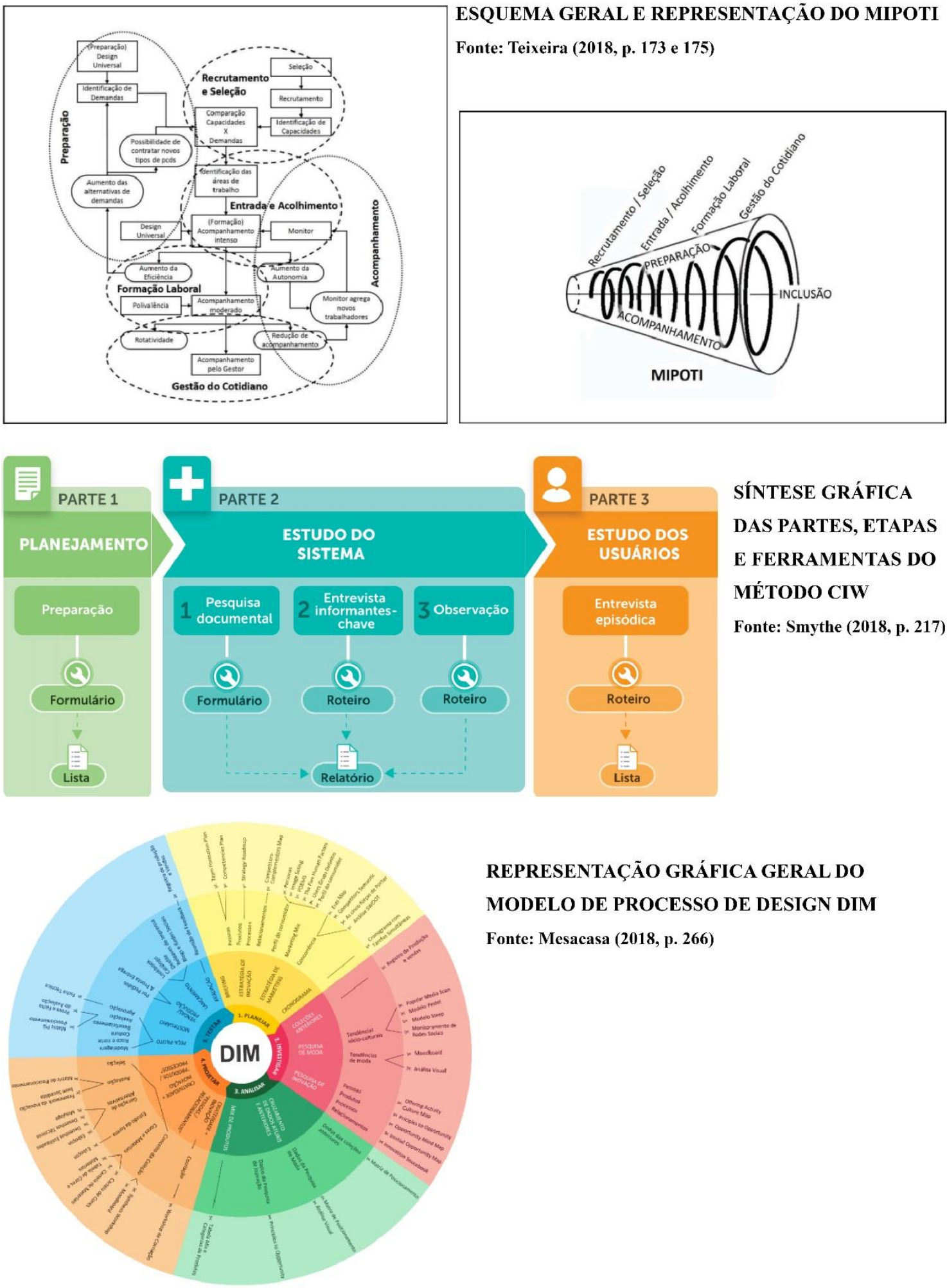

\section{REPRESENTAÇÃO GRÁFICA GERAL DO MODELO DE PROCESSO DE DESIGN DIM}

Fonte: Mesacasa (2018, p. 266)

Figura 3: Artefatos resultantes das pesquisas analisadas. Adaptado pelos autores.

Em consonância com as características da DSR, todas as teses apresentaram abordagem qualitativa e natureza aplicada. Com relação aos objetivos, as pesquisas foram classificadas por seus autores como exploratórias ou prescritivas. A principal característica da DSR, contudo, diz respeito à utilização da lógica abdutiva, típica do design, que postula como algo poderia ser (DRESCH; LACERDA; ANTUNES JÚNIOR, 2015; MARTIN, 2010). As três teses adotaram 
essa lógica na elaboração dos artefatos, mas Smythe (2018) incorporou também a perspectiva indutiva ${ }^{2}$, aplicada na observação do fenômeno, realizada por meio de estudos de caso (Etapa 1), enquanto Mesacasa (2018) empregou a dedução ${ }^{3}$ para avaliar o artefato por meio de grupos focais. Quanto à operacionalização da DSR, todos os trabalhos analisados adotaram como referência Dresch, Lacerda e Antunes Júnior (2015), cujo método apresenta caráter iterativo, como destacado por Smythe (2018) e Teixeira (2018). Os autores das três teses, contudo, estruturaram seus métodos de maneiras diversas, como demonstra a Figura 4.
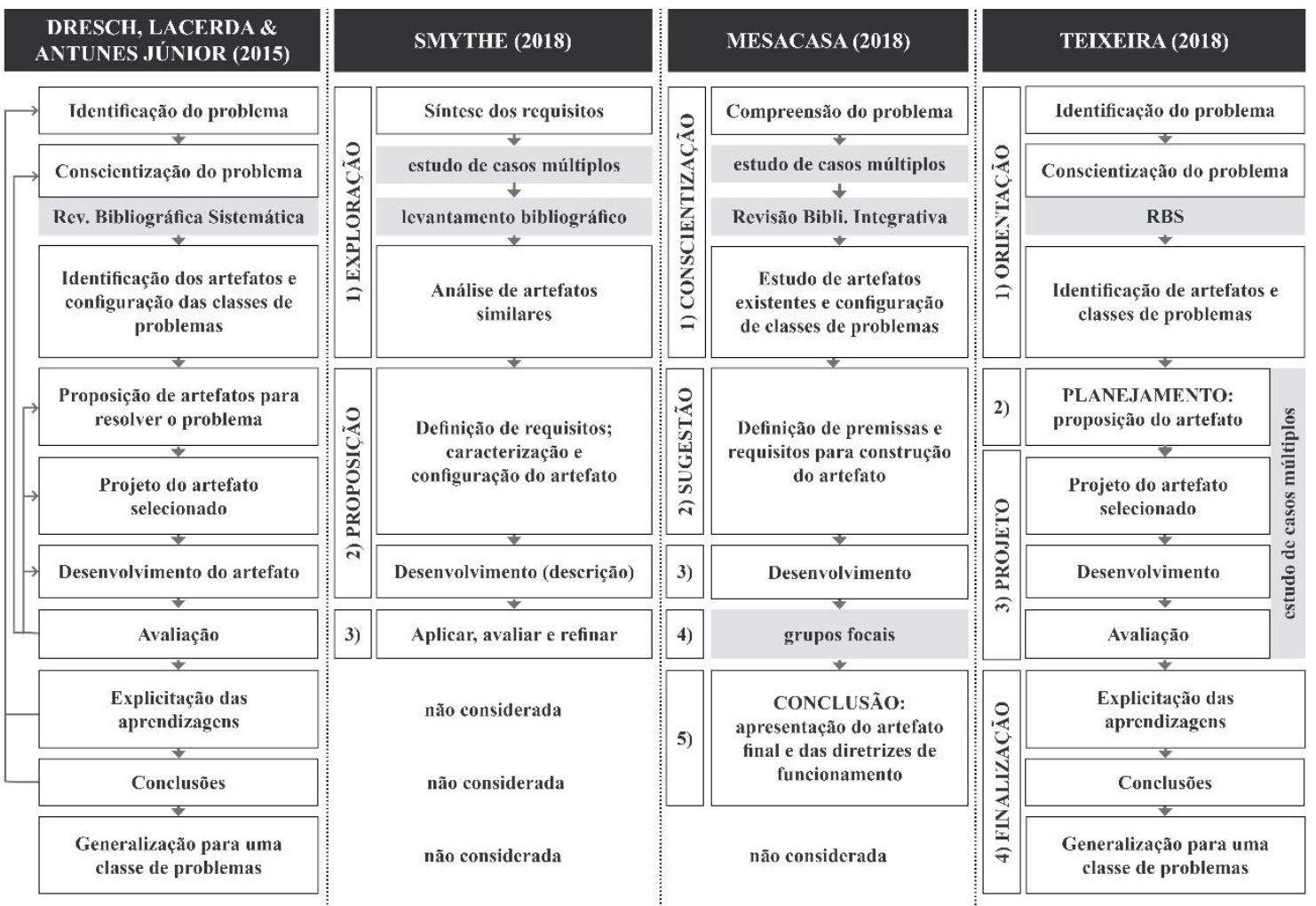

Figura 4: Correspondência entre etapas e atividades da DSR nas teses analisadas. Elaborado pelos autores.

As três teses apresentam concordância na definição das atividades que constituem a primeira etapa de seus métodos, embora utilizem diferentes nomenclaturas. A análise das teses indica ser esse o momento ideal para empregar outros métodos combinados à DSR. Os três pesquisadores cujos trabalhos foram analisados utilizaram a pesquisa bibliográfica e apontaram sua importância para a fundamentação das soluções desenvolvidas. Outro método empregado nessa etapa foi o estudo de caso, que envolveu estudo de campo (SMYTHE, 2018) e levantamento com usuários por meio de entrevistas e questionário em escala Likert de 1 a 6 (TEIXEIRA, 2018). Esses procedimentos auxiliares favorecem a conscientização do problema e permitem a obtenção de outros resultados além do artefato, como construção de conhecimento e contribuições teóricas (HEVNER et al., 2004; DRESCH; LACERDA; ANTUNES JÚNIOR, 2015; SMYTHE, 2018; MESACASA, 2018).

\footnotetext{
2 Parte do específico para o geral, postulando o que é eficiente (DRESCH; LACERDA; ANTUNES JÚNIOR, 2015; MARTIN, 2010).

${ }^{3}$ Parte do geral para o específico, postulando como algo deve ser (Ibidem).
} 
Ainda sobre a primeira etapa das pesquisas, cabe destacar as inconsistências identificadas em relação ao protocolo de Dresch, Lacerda e Antunes Junior (2015), utilizado nos três casos como referência. Teixeira (2018), por exemplo, não apresenta as classes de problemas, ainda que essa atividade esteja prevista em sua tese, e não explicita a identificação de artefatos como tal, embora apresente, na fundamentação teórica, diferentes métodos similares ao por ele desenvolvido. Mesacasa (2018), por sua vez, analisa diferentes modelos de processos de design no início da seção de "desenvolvimento" da tese, como resultado da identificação de artefatos. As classes de problemas foram configuradas a partir dessa identificação; contudo, foram estruturadas de tal modo que mais classificam os tipos de artefatos do que os problemas solucionados. Já Smythe (2018), ao invés de identificar artefatos similares ao que seria desenvolvido, apresentou uma descrição e classificação geral de artefatos no âmbito da design science. A classe de problemas foi estruturada com foco no escopo do método desenvolvido; porém, a pesquisadora indicou não as possíveis soluções para a classe de problema identificada, mas seus atributos. Talvez essas inconsistências devam-se ao fato de não haver "uma definição conceitual da classe de problemas ou uma proposição para sua construção" (LACERDA et al., 2013, p. 747), sendo as classes de problemas vagamente definidas na literatura sobre DSR como "a organização de um conjunto de problemas práticos ou teóricos que contenha artefatos úteis para a ação nas organizações" (DRESCH; LACERDA; ANTUNES JÚNIOR, 2015, p. 104).

Ao final da primeira etapa, as diferentes estratégias de pesquisa adotadas permitiram a definição de diretrizes, premissas ou requisitos a partir dos quais os três pesquisadores propuseram os artefatos que foram, então, desenvolvidos na segunda etapa. Apesar de essa ser uma etapa essencial do método, os procedimentos referentes ao processo de desenvolvimento não foram propriamente explicitados nas teses. Igualmente, a literatura sobre DSR não elucida como construir o artefato. Assim, o que se observou nas teses foi a descrição dos elementos que constituem os artefatos desenvolvidos e a justificativa das escolhas e decisões envolvidas.

$\mathrm{Na}$ etapa de avaliação, os três pesquisadores adotaram diferentes procedimentos. Mesacasa (2018) avaliou o modelo desenvolvido em sua tese por meio da condução de grupos focais. Já Teixeira (2018) e Smythe (2018) realizaram eles mesmos a avaliação dos métodos desenvolvidos. Enquanto Teixeira (2018) apenas discutiu sua viabilidade, utilizando como referencial os dados coletados durante o estudo de caso, Smythe (2018) aplicou seu método em dois contextos reais diferentes, adotando uma abordagem iterativa que permitiu o refinamento do método proposto. Cabe destacar que a literatura sobre DSR é vaga com relação aos procedimentos avaliativos. Hevner et al. (2004) listam algumas possíveis formas de avaliação (observacional, analítica, descritiva, experimental e teste), mas as descrições são sucintas e pouco elucidativas.

Com relação ao rigor das pesquisas, fator relevante para diferenciar a DSR da prática do design e estabelecê-la como método científico (HEVNER et al., 2004; DRESCH; LACERDA; ANTUNES JÚNIOR, 2015), ressalta-se que nenhuma das teses apresentou um protocolo da DSR em si, mas apenas protocolos relacionados aos métodos tradicionais utilizados. Devido à diversidade de métodos, procedimentos e protocolos adotados às vezes em uma mesma pesquisa, a compreensão da estratégia de realização das pesquisas analisadas é, em alguns momentos, uma tarefa difícil, o que pode dificultar a replicação bem sucedida por outros investigadores. 
Também se observou que algumas atividades essenciais à DSR, incluídas no protocolo de Dresch, Lacerda e Antunes Júnior (2015), não foram realizadas pelos pesquisadores. Por exemplo, em nenhuma tese foram identificadas as heurísticas ${ }^{4}$ relacionadas aos artefatos desenvolvidos. $\mathrm{Na}$ quarta etapa, também não foi explicitada a generalização para uma classe de problemas. Contudo, os métodos e o modelo desenvolvidos como parte das teses destinam-se a ser aplicados por outros designers e pesquisadores, fato que possibilita a ampliação dos resultados para contexto similares aos considerados pelos pesquisadores.

Com relação à eficácia da DSR para o desenvolvimento das pesquisas de doutoramento, Mesacasa (2018) destaca a sua operacionalidade, devido a estar estruturada de tal modo que as etapas subsequentes são abastecidas pelos resultados das anteriores. Já Smythe (2018) ressalta como contribuições da DSR a reflexão crítica proporcionada ao longo do processo de pesquisa. A pesquisadora também discorre, na conclusão de sua tese, sobre a correspondência do método com a prática de design, a qual facilitou a "compreensão sobre as saídas desejadas, ou seja, como se daria a construção da solução" (Ibidem, p. 226). Por fim, destaca-se que os pesquisadores, ao traçarem suas considerações sobre o método, não indicaram desafios relativos à DSR em si, apontando apenas dificuldades encontradas para obter a colaboração de terceiros durante a pesquisa (e.g. TEIXEIRA, 2018; MESACASA, 2018).

\section{Action Design Research: Relato de experiência}

A experiência aqui relatada tratou da aplicação de um método híbrido baseado no paradigma da design science. O objetivo da pesquisa era identificar e sistematizar heurísticas para a aplicação dos princípios do open design ao desenvolvimento de vestuário mais sustentável, com utilização predominante de tecnologias de fabricação digital. As heurísticas foram identificadas por meio do desenvolvimento de produtos, envolvendo parceria com diversos cocriadores e duas empresas, motivo pelo qual optou-se por conduzir uma pesquisa-ação sob o paradigma da design science. $\mathrm{O}$ método adotado foi a Action Design Research (ADR), cujas principais diferenças em relação à DSR são o caráter cíclico de sua aplicação, baseado nos ciclos de Hevner (2007), e a concomitância entre desenvolvimento e avaliação de artefatos.

A adequação do método à proposta de pesquisa baseou-se nos procedimentos metodológicos de Sein et al. (2011), Hevner (2007) e Dresch, Lacerda e Antunes Júnior (2015). A pesquisa apresentou caráter exploratório-descritivo e envolveu quatro etapas: 1) Problematização, 2) Desenvolvimento, 3 ) Avaliação e 4) Formalização da Aprendizagem. A Figura 5 sintetiza a estratégia de realização da pesquisa. No centro da imagem, destaca-se que foram empregadas diferentes lógicas, sendo a principal delas a abdutiva. Outra característica importante foi a adoção alternada dos pensamentos divergente e convergente, representada pelo modelo do processo de design conhecido como Double Diamond (DESIGN COUNCIL, 2015).

\footnotetext{
${ }^{4}$ A DSR envolve a identificação de heurísticas de construção e contingencias. As heurísticas de construção correspondem à descrição da organização interna do artefato. Já as heurísticas contingenciais caracterizam a relação com o contexto de utilização, explicitando os limites e as condições de utilização (Dresch; Lacerda; Antunes Júnior, 2015).
} 


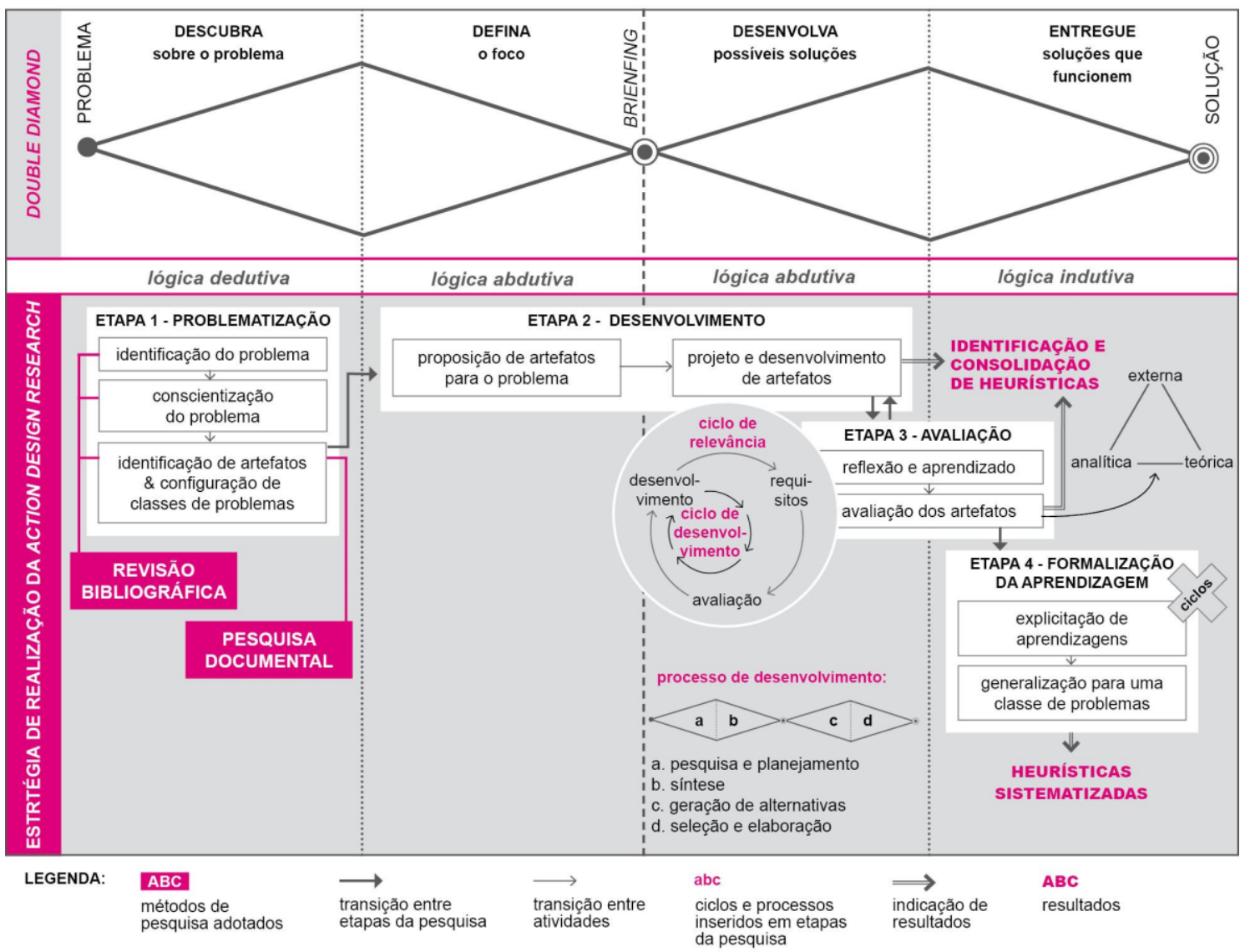

Figura 5: Estratégia de realização da Action Design Research. Fonte: Perez (2018, p. 144)

Um dos desafios da pesquisa foi a configuração de classes de problemas, o qual foi resolvido por meio da elaboração de um quadro com três colunas principais: 1) classes de problemas, com os principais elementos relacionados ao tema central da investigação; 2) soluções, que podem ser subdivididas em duas colunas, e 3) referências. Após, foi realizada pesquisa documental para a identificação e análise de artefatos já existentes para a solução das classes de problemas mapeadas por meio de revisão bibliográfica sistemática e assistemática. Embora Dresch, Lacerda e Antunes Júnior (2015) recomendem realizar essa atividade a partir da revisão bibliográfica, a pesquisa documental mostrou-se necessária, pois poucos artefatos foram encontrados na literatura. Como resultado dessa análise, foram detectadas e sistematizadas as heurísticas associadas aos artefatos identificados. Aqui, a principal dificuldade foi compreender como identificar e sistematizar as heurísticas, sendo necessário recorrer a outras referências bibliográficas (e.g., SILVEIRA, 2016), uma vez que a literatura sobre design science consultada não esclarecia como formular as heurísticas. Assim, as heurísticas identificadas por meio da revisão bibliográfica e da pesquisa documental foram sistematizadas como ilustrado na Figura 6. 

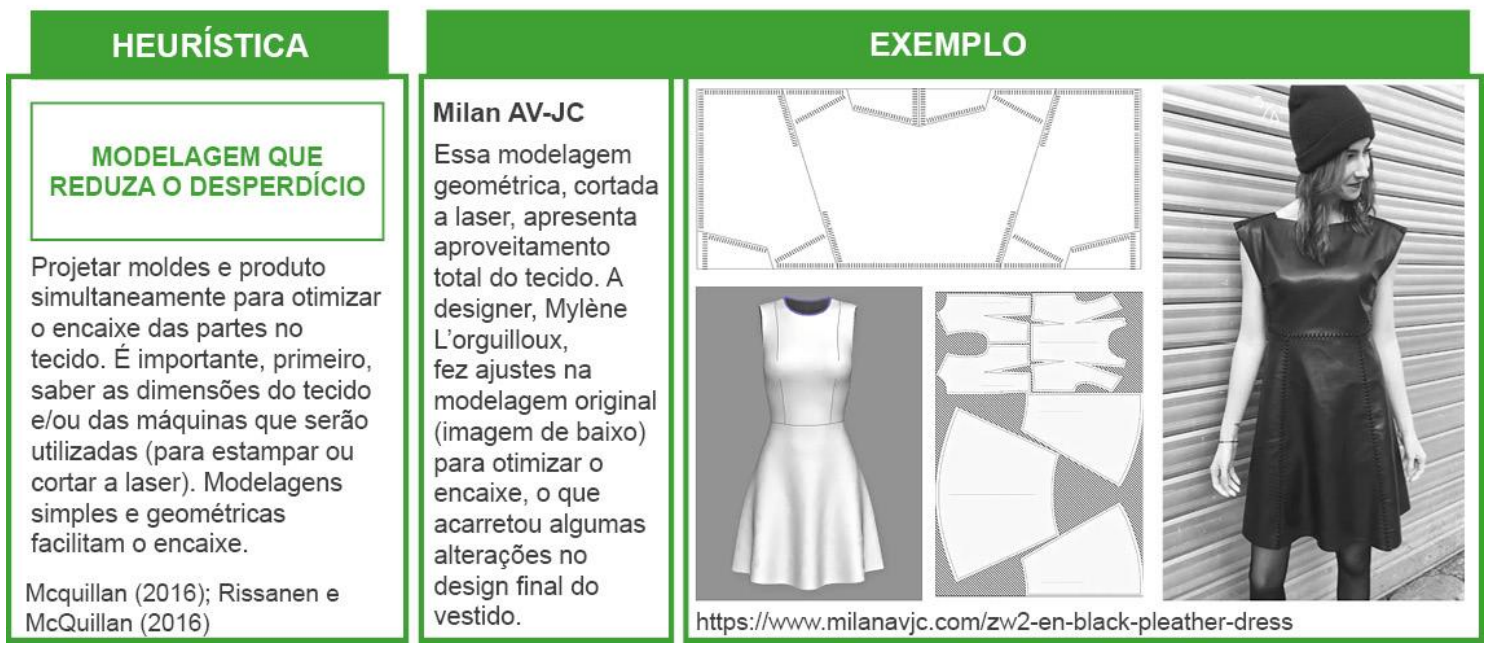

Figura 6: Uma das heurísticas formuladas a partir da revisão bibliográfica e da pesquisa documental. Fonte: Perez (2018, p. 170)

A segunda e a terceira etapas, Desenvolvimento e Avaliação de artefatos, foram conduzidas de maneira iterativa, adotando-se os ciclos apontados por Hevner (2007): o próprio ciclo de desenvolvimento e o que o autor chama de ciclo de relevância (relevance cycle). Esses dois ciclos são interdependentes, embora preservem relativa independência durante sua execução (HEVNER, 2007). O ciclo de desenvolvimento, como o nome sugere, ocorre durante o processo de desenvolvimento de artefatos e está inserido no ciclo de relevância, que é mais amplo e se inicia com o desenvolvimento, estendendo-se à etapa de avaliação. Na estratégia de realização da pesquisa aqui relatada, os ciclos de relevância permitiram a consolidação das heurísticas.

Foi inicialmente planejada a condução de no mínimo dois ciclos de relevância, um para a definição de heurísticas e um segundo para a sua consolidação. O primeiro ciclo ocorreu no contexto de uma disciplina eletiva semestral, com participação de 15 alunos de graduação em Design de Produto da UFPR, os quais dividiram-se em cinco equipes para o desenvolvimento de artefatos. Como alvo dos produtos desenvolvidos, participou também deste ciclo uma empresa de moda holandesa que trabalha com open design. Para a realização do segundo ciclo de relevância, foi firmada parceria com uma marca de moda local e promovido um projeto de cocriação, com chamada aberta para a participação de estudantes de áreas criativas e novos designers.

Durante os dois ciclos, a etapa de desenvolvimento foi apenas planejada e propulsionada pela pesquisadora, que adotou o papel de observadora participante, sendo os artefatos desenvolvidos pelos participantes da pesquisa. O processo de design foi delimitado de acordo com referências de design e moda pertinentes ao escopo do trabalho. Em todos os ciclos de relevância, a etapa de avaliação dos artefatos ocorreu por meio da aplicação de três estratégias: avaliação externa (especialistas, público ou grupos focais), analítica e teórica. Planejar essa etapa foi um dos desafios enfrentados, sendo necessário recorrer à literatura sobre design para coletar procedimentos que pudessem ser úteis. Também foram desenvolvidos alguns procedimentos próprios, como a elaboração de tabelas para confrontar as heurísticas adotadas durante o desenvolvimento com aquelas levantadas quando da identificação e análise de artefatos similares. 
Como já mencionado, inicialmente foram planejados dois ciclos de relevância. Ao final do segundo, contudo, foi identificada a necessidade de dar início a um terceiro ciclo, pois os artefatos desenvolvidos não atendiam aos requisitos de projeto previamente estabelecidos. Considerando as dificuldades enfrentadas durante o segundo ciclo para obter o envolvimento dos participantes da pesquisa, além da necessidade de o novo ciclo ser alimentado pelos resultados dos ciclos anteriores, optou-se por alterar o papel da pesquisadora, que tornou-se também cocriadora dos artefatos. Desse modo, foi possível não apenas garantir o atendimento aos requisitos de projeto, como aplicar as heurísticas anteriormente identificadas. Finalizada a avaliação do terceiro ciclo de relevância, teve início a última etapa da pesquisa, Formalização da Aprendizagem. A principal atividade, nesse momento, foi a sistematização e apresentação das heurísticas consolidadas ao longo dos três ciclos (Figura 7), sendo cada heurística generalizada para uma classe de problemas.

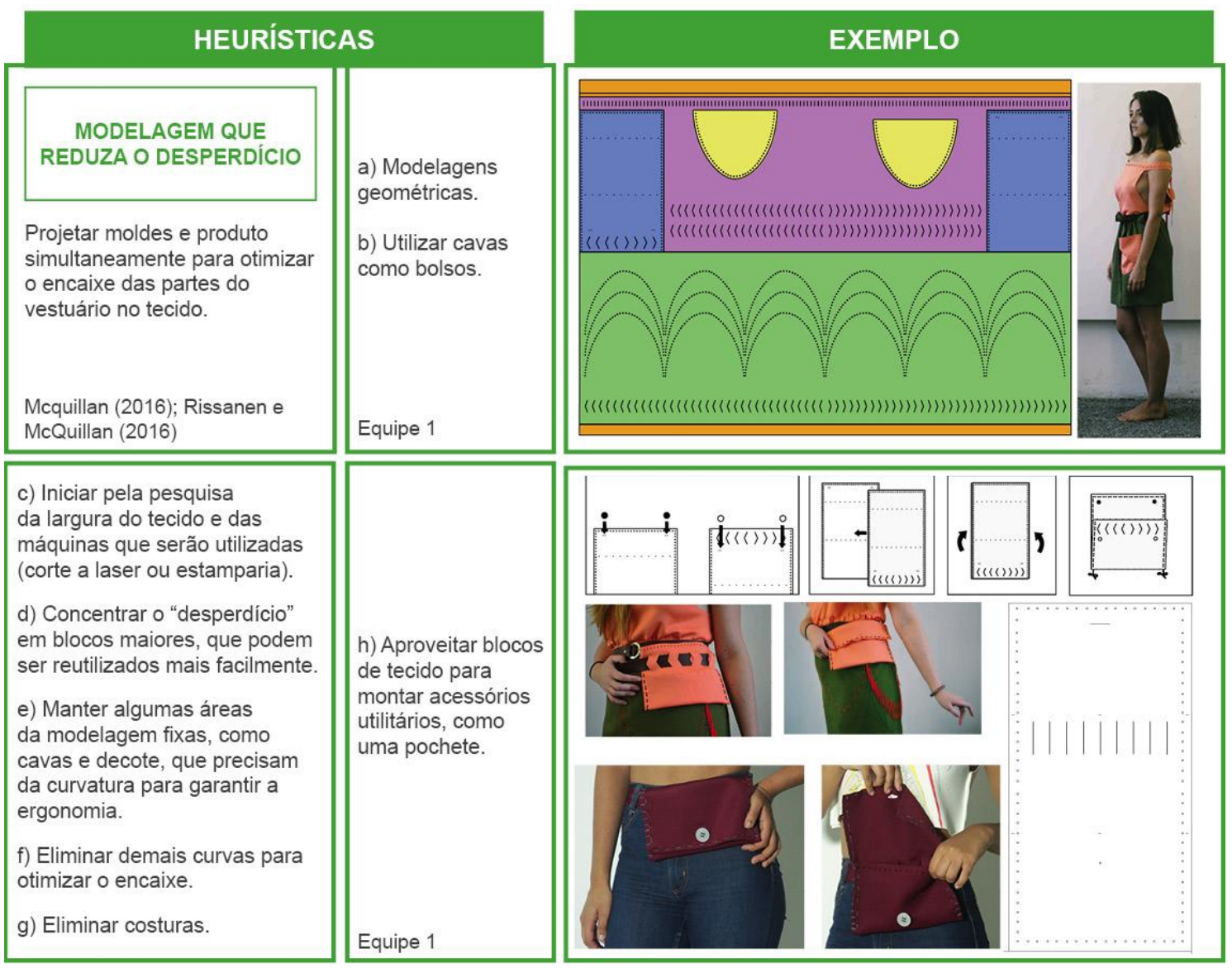

Figura 7: Heurística da classe “sustentabilidade”, consolidada após a segunda e terceira etapas da pesquisa. Fonte: Perez (2018, p. 312)

A abordagem híbrida adotada como estratégia de realização da pesquisa tornou difícil a categorização do método, que não se encaixa em preceitos tradicionais de pesquisa científica. A própria descrição do método foi desafiadora, motivo pelo qual foi estruturado um protocolo para a ADR. Apesar da complexidade e da dificuldade em descrevê-lo ou categorizá-lo, o método apresentou diversas contribuições para a construção do conhecimento em design, possibilitando a geração de soluções prescritivas (nesse caso, as heurísticas), que podem ser utilizadas como referência por outros designers ou pesquisadores para o avanço da prática e da pesquisa relativa ao open design. Ademais, a experiência de conduzir uma pesquisa a partir do paradigma da design 
science contribuiu para a formação conjunta de uma pesquisadora-designer, pois foram aplicados e adquiridos conhecimentos tanto relativos à pesquisa científica quanto à prática do design.

\section{Considerações finais}

A pesquisa científica em design pode ser conduzida de diferentes formas, com propósitos diversos. Uma das possibilidades é a condução de pesquisas através do design, as quais correspondem à abordagem da design science. Como a Revisão Bibliográfica Sistemática revelou, a design science tem sido citada por diversos trabalhos nacionais desde 2010. É ainda uma abordagem científica nova e pouca conhecida, mas que tem despertado interesse crescente em áreas como engenharia e administração. Paradoxalmente, a design science é pouco empregada no design. Contudo, espera-se que essa realidade mude em breve a partir das recentes teses e dissertações em design que adotaram métodos da design science.

O presente artigo identificou e analisou três teses e uma dissertação em design que empregam os métodos Design Science Research (DSR) e Action Design Research (ADR). Por meio da análise das teses encontradas e de um relato de experiência, mostrou-se que os métodos da design science podem ser utilizados para o desenvolvimento de diferentes artefatos e soluções prescritivas, sendo adaptáveis e podendo incluir outros procedimentos metodológicos. Quanto às contribuições dos métodos analisados, evidencia-se que seus procedimentos favorecem a relevância da pesquisa e possibilitam a construção de conhecimento ao longo de todo o processo. Contudo, são necessárias mais referências de base para permitir planejamento e descrição rigorosos dos procedimentos metodológicos, sobretudo quanto a: configuração de classes de problemas; desenvolvimento e avaliação dos artefatos; identificação e consolidação de heurísticas.

A realização de pesquisas sob o paradigma da design science pode ser enriquecedora tanto para a ampliação do campo epistemológico do design e das possibilidades de atuação dos designers, quanto para a formação dos próprios pesquisadores-designers que as conduzem. Assim, espera-se que este artigo instigue mais pesquisadores do design a se familiarizarem com os métodos da design science e a adotarem-nos quando apropriado, sugerindo-se que estabeleçam pensamento crítico a respeito desses métodos, indicando suas forças e fragilidades, de modo a contribuir para o aprimoramento dessa abordagem de pesquisa focada na área do design.

\section{Referências}

BONSIEPE, G. Design, cultura e sociedade. São Paulo: Blucher, 2011.

CONFORTO, E. C.; AMARAL, D. C.; SILVA, S. L. DA. Roteiro para revisão

bibliográfica sistemática: aplicação no desenvolvimento de produtos e gerenciamento de projetos. Trabalho apresentado no $8^{\circ}$ Congresso Brasileiro de Gestão de Desenvolvimento de Produto, Porto Alegre, 2011.

CROSS, N. Designerly ways of knowing: design discipline versus design science. Design

Issues, v. 17, n. 3, p. 49-55, 2001. 
DESIGN COUNCIL. The Design Process: What is the Double Diamond? 2015. Disponível em: <http://www.designcouncil.org.uk/news-opinion/design-process-what-doublediamond>. Acesso em: 05 nov. 2017.

DRESCH, A.; LACERDA, D. P.; ANTUNES JUNIOR, J. A. V. Design Science research: método de pesquisa para avanço da ciência e tecnologia. Porto Alegre: Bookman, 2015.

FRAYLING, C. Research in Art and Design. Royal College of Art Research Papers, v. 1, n. 1, 1993.

HEVNER, A. R. A Three Cycle View of Design Science Research. Scandinavian Journal of Information Systems, v. 19, n. 2, p. 87-92, 2007.

HEVNER, A. R.; MARCH, S. T.; PARK, J.; RAM, S. Design Science in Information Systems Research. MIS Quarterly, v. 28, n. 1, p. 75-105, 2004.

LACERDA, D. P.; DRESCH, A.; PROENÇA, A.; ANTUNES JÚNIOR, J. A.V. Design Science Research: método de pesquisa para a engenharia de produção. Gestão \& Produção, v. 20, n. 4, p. 741-761, 2013.

MANZINI, E. Design, when everybody designs: an introduction to design for social innovation. Traduzido por: COAD, R. Massachusetts: MIT Press.

MARCH, S. T.; SMITH, G. F. Design and natural science research on information technology. Decision Support Systems, v. 15, n. 4, p. 251-266, 1995.

MARTIN, R. Design de negócios: por que o design thinking se tornará a próxima vantagem competitiva dos negócios e como se beneficiar disso. Rio de Janeiro: Elsevier, 2010.

MESACASA, A. DIM - Design Inova Moda: modelo de processo de design com ênfase na inovatividade para o setor do vestuário. 357 f. Tese (Doutorado em Design) - Universidade Federal do Paraná, Curitiba, 2018.

PEREZ, I. U. Open design na promoção de economias distribuídas: heurísticas para o desenvolvimento do vestuário. 2018. 371 f. Dissertação (Mestrado em Design) Universidade Federal do Paraná, Curitiba, 2018.

SANTOS, A. dos. Design Science. In: (Ed.). Seleção do método de pesquisa: guia para pós-graduandos em design e áreas afins. Curitiba: Insight, 2018.

SEIN, M. K.; HENFRIDSSON, O.; PURAO, S.; ROSSI, M.; LINDGREN, R. Action design research. MIS Quarterly, v. 30, n. 3, p. 611-642, 2011.

SILVA, T. B. P. E. Um campo epistemológico para o Design. Revista de Design, Tecnologia e Sociedade, v. 2, n. 2, p. 23-41, 2015.

SILVEIRA, E. L. Indexação e Mapeamento de Exemplos para as Heurísticas Compiladas da TRIZ. 131 f. Dissertação (Mestrado em Engenharia Mecânica) Universidade Tecnológica Federal do Paraná, Curitiba, 2016.

SIMON, H. A. The Sciences of the Artificial. 3. ed. Cambridge: MIT Press, 1996.

SMYTHE, K. C. A. DA S. Proposta de método de obtenção de dados sobre comportamento informacional dos usuários no processo de wayfinding em ambientes hospitalares. 289 f. Tese (Doutorado em Design) - Universidade Federal do Paraná, Curitiba, 2018.

TEIXEIRA, E. S. M. Design universal: método de inclusão de pessoas com deficiência em postos de trabalho de produção industrial. 216 f. Tese (Doutorado em Design) - Universidade Federal do Paraná, Curitiba, 2018. 


\section{Sobre os autores:}

\section{Iana Uliana Perez}

Doutoranda em Design (UNESP - Universidade Estadual Paulista), Mestra em Design (UFPR Universidade Federal do Paraná) e graduada em Design de Moda (UEL - Universidade Estadual de Londrina). Atualmente é membro e pesquisadora do Laboratório e Grupo de Pesquisa em Design Contemporâneo: sistemas, objetos e cultura (UNESP) e do Grupo de Pesquisa em Design, Sustentabilidade e Inovação (UEL).

iana.uli@gmail.com

\section{Mônica Cristina de Moura}

Coordenadora do Laboratório de Pesquisa em Design Contemporâneo e líder do Grupo de Pesquisa Design Contemporâneo: sistemas, objetos e cultura (CNPq/UNESP). Professora do Departamento de Design e Orientadora no Programa de Pós-graduação em Design da UNESP. Realizou pós-doutorado na Pontifícia Universidade Católica (PUC-Rio) e na Universidade do Minho. Doutora e Mestre em Comunicação e Semiótica (PUC-SP).

monica.moura@unesp.br

\section{Fausto Orsi Medola}

Professor no Departamento de Design e vice coordenador do Programa de Pós-graduação em Design da UNESP. Doutor em Bioengenharia (USP) com estágio de doutorado sanduíche na Georgia Institute of Technology (Atlanta, EUA). Mestre em Bioengenharia (USP) e graduado em Fisioterapia (UEL). É líder do Grupo de Pesquisa Design e Tecnologia Assistiva e pesquisador no GP Desenho Industrial: Projeto e Interfaces.

fausto.medola@unesp.br 